\title{
Trigonometric Cubic B-spline Collocation Method for Solitons of the Klein-Gordon Equation
}

\author{
Alper Korkmaz ${ }^{1, *}$, Ozlem Ersoy ${ }^{2}$ and Idiris Dag ${ }^{3}$

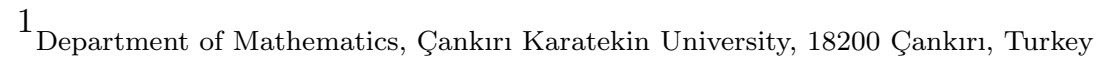 \\ 2 Department of Mathematics \& Computer, Eskisehir Osmangazi University, 26480 Eskisehir, Turkey; \\ ozersoy@ogu.edu.tr \\ 3 Department of Computer Engineering, Eskisehir Osmangazi University, 26480 Eskisehir, Turkey; \\ idag@ogu.edu.tr \\ $*_{\text {Correspondence: akorkmaz@karatekin.edu.tr }}$
}

December 2, 2016

\begin{abstract}
In the present study, we derive a new B-spline technique namely trigonometric B-spline collocation algorithm to solve some initial boundary value problems for the nonlinear Klein-Gordon equation. In order to carry out the time integration with Crank-Nicolson implicit method, the order of the equation is reduced to give a coupled system of nonlinear partial differential equations. The collocation approximation based on trigonometric cubic B-splines for spatial discretization is followed by the linearization of the nonlinear term. The efficiency and accuracy of the present method are validated by measuring the error between the numerical and analytical solutions when exist. The conservation laws representing momentum and energy are also computed for all problems.
\end{abstract}

Keywords: Klein-Gordon equation; trigonometric cubic B-spline; collocation; soliton; wave motion; conservation laws

AMS2010: 65M70.

\section{Introduction}

Consider the Klein-Gordon equation of the form

$$
u_{t t}-u_{x x}+P^{\prime}(u)=0
$$


where $u=u(x, t), P^{\prime}(u)$ is some reasonable nonlinear function of $u$ [1. $P(u)$ does not include the derivatives of $u$ and can be chosen as the potential energy in many studies. The equation was derived by Klein and Gordon as a relativistic equation for a charged particle in an electromagnetic field [2]. Some particular forms of the equation are used as model for many fields such as Josephson junction transmission lines, dislocation in crystals, the propagation in ferromagnetic materials of waves carrying rotations of the direction of magnetization, laser pulses in two state media, torsional waves propagating down a stretched wire in a pendula system, etc. According to Detweiler, The Klein-Gordon equation can also be useful to analyze the rotating black holes [3]. The equation can be constructed geometrically by the invariance of the scalar curvature with some particular gauge and coordinate transformations [4]. The scattering field theory for the Klein-Gordon equation was developed by following the usual procedure of a first order equivalent equation in the vector valued functions Hilbert space equipped with a finite energy norm [5]. The spectral and scattering theory based on the eigenfunction expansion was also derived for the Klein-Gordon equation in the static external field [6].

Besides, its applications in many fields summarized above, the Klein-Gordon equation with cubic nonlinear term can be converted to the nonlinear Schrï $i \frac{1}{2}$ dinger equation with cubic nonlinearity [7]. However, the sign of the cubic term is the key for the existence of the envelope soliton or hole solutions owing to the fact that the stability of the nonlinear Klein-Gordon equation depends on it [8].

The existence of compactonlike kink solutions of the Klein-Gordon equation was discussed by investigating the existence and stability of compact entities numerically [9]. Kim and Hong [10] determine the solitary wave solutions including the powers of sech and tanh functions of the generalized Reaction Duffing equations, which is a generalization of some nonlinear equations, the Landau-Ginzburg-Higgs, the $\Phi^{4}$ and the Klein-Gordon equation with cubic nonlinear term, by the auxiliary function method. In a similar study, the positive and negative frequency planewave solutions developed from the solutions of some field equations [11. The extended first kind elliptic sub equation method is an alternative way to generate the exact traveling wave solutions including bell and kink shaped solitary wave, periodic and singular solutions [12]. Some more exact solutions including some hyperbolic and trigonometric functions have also been developed by modified simple equation method that uses homogenous balance between the highest order linear and the nonlinear terms [13]. In another interesting approach to the solutions of the Klein-Gordon equation, Chambers [14] derived the solutions from solutions of the wave equation. Bound state type solutions of the Klein-Gordon equation were constructed for various potentials [15]. Burt and Reid [16] set up the exact formal solution of the nonlinear Klein-Gordon equation from the solutions of the linear one. They also discussed about the generalization of a second order ordi- 
nary differential equation satisfied by a particular homogenous function can lead to the nonlinear Klein-gordon equation. Interaction of solitons of the Klein-Gordon equation was investigated in different aspects in various numerical studies [17 19]. Different from many analytical studies summarized above, various numerical algorithms have also been developed to obtain solutions of different problems for the Klein-Gordon equation. The central second difference approximation of the finite difference method was implemented to the nonlinear Klein-Gordon equation to solve some initial boundary value problems to show the bounded solutions as $t \rightarrow \infty[20]$. The degree of the power term directly affects the amplitude and the number of oscillations. Jimï $; \frac{1}{2}$ nez and Vï $\frac{1}{2}$ zquez $[21]$ compared the results obtained by four explicit finite difference schemes and concluded that the energy conserving scheme is the most convenient technique to integrate the Klein-Gordon equation to examine the long time behaviour. Some initial boundary value problems for the inhomogenous form of the Klein-Gordon equation with various degreed nonlinear terms were also solved by the thin plate splines radial basis functions collocation method [22]. Multiquadric Quasi-interpolation technique is another efficient technique to determine the solutions of the equation [23]. Cao and Guo 24] proposed the Fourier collocation method to solve a periodic problem by investigating its stability and convergence properties.

Classical polynomial cubic B-spline collocation on a uniform mesh distribution was implemented to the some problems derived on the nonlinear Klein-Gordon equation [25]. Zahra et al. 26] derived an unconditionally stable collocation method based on the cubic B-splines for the solution of an initial boundary value problem. Different from these studies, we suggest a new collocation method based on non polynomial cubic B-spline, namely trigonometric cubic B-splines, to solve some initial boundary value problems for the nonlinear Klein-Gordon equation of the particular form

$$
u_{t t}-u_{x x}=\varepsilon_{1} u+\varepsilon_{2} u^{3}
$$

where $\varepsilon_{1}$ and $\varepsilon_{2}$ are nonzero constants. Assuming $v=u_{t}$ reduces the order of the equation to lead a coupled system of nonlinear partial differential equations

$$
\begin{aligned}
& v_{t}=u_{x x}+\varepsilon_{1} u+\varepsilon_{2} u^{3} \\
& u_{t}=v
\end{aligned}
$$

The complementary conditions describing the associated problem are

$$
\begin{aligned}
& u(x, 0)=f(x), a \leq x \leq b \\
& u_{x}(a, t)=u_{x}(b, t)=0, t>0 \\
& v_{x}(a, t)=v_{x}(b, t)=0, t>0
\end{aligned}
$$

in the artifical problem interval $[a, b]$. 


\section{Numerical Approach and Construction of the Algorithm}

The discretization of the system (3) in time by usual forward finite difference and by Crank-Nicolson method yields

$$
\begin{aligned}
& \frac{v^{n+1}-v^{n}}{\Delta t}=\frac{u_{x x}^{n+1}+u_{x x}^{n}}{2}+\varepsilon_{1} \frac{u^{n+1}+u^{n}}{2}+\varepsilon_{2} \frac{\left(u^{3}\right)^{n+1}+\left(u^{3}\right)^{n}}{2} \\
& \frac{u^{n+1}-u^{n}}{\Delta t}=\frac{v^{n+1}+v^{n}}{2}
\end{aligned}
$$

where $u^{n+1}=u(x,(n+1) \Delta t)$ represents the solution at the $(n+1)$ th. time level. Here $t^{n+1}=t^{n}+\Delta t$, and $\Delta t$ is the time step, superscripts denote the time levels. Linearization of the term $\left(u^{3}\right)^{n+1}$ in (5) as

$$
\left(u^{3}\right)^{n+1}=3 u^{n+1}\left(u^{2}\right)^{n}-2\left(u^{3}\right)^{n}
$$

gives the time-integrated Klein-Gordon equation

$$
\begin{aligned}
& \frac{v^{n+1}-v^{n}}{\Delta t}=\frac{u_{x x}^{n+1}+u_{x x}^{n}}{2}+\varepsilon_{1} \frac{u^{n+1}+u^{n}}{2}+\varepsilon_{2} \frac{3 u^{n+1}\left(u^{2}\right)^{n}-\left(u^{3}\right)^{n}}{2} \\
& \frac{u^{n+1}-u^{n}}{\Delta t}=\frac{v^{n+1}+v^{n}}{2}
\end{aligned}
$$

Let $G$ be a uniform grid distribution of the artificial problem interval $[a, b]$ defined as $G: a=x_{1}<x_{2}<\ldots<x_{N}=b, h=x_{i}-x_{i-1}, i=2,3, \ldots, N$. Different from the other B-splines 27-30], the trigonometric cubic B-splines with nonzero values in this interval for this grid distribution are defined as

$$
\begin{aligned}
C_{i}(x) & =\frac{1}{\theta}\left\{\begin{array}{lcc}
\omega^{3}\left(x_{i-2}\right) & , & {\left[x_{i-2}, x_{i-1}\right]} \\
\omega\left(x_{i-2}\right)\left(\omega\left(x_{i-2}\right) \phi\left(x_{i}\right)+\omega\left(x_{i-1}\right) \phi\left(x_{i+1}\right)\right)+\phi\left(x_{i+2}\right) \omega^{2}\left(x_{i-1}\right) & , & {\left[x_{i-1}, x_{i}\right]} \\
\omega\left(x_{i-2}\right) \phi^{2}\left(x_{i+1}\right)+\phi\left(x_{i+2}\right)\left(\omega\left(x_{i-1}\right) \phi\left(x_{i+1}\right)+\omega\left(x_{i}\right) \phi\left(x_{i+2}\right)\right) & , & {\left[x_{i}, x_{i+1}\right]} \\
\phi^{3}\left(x_{i+2}\right) & , & {\left[x_{i+1}, x_{i+2}\right]} \\
0 & , & \text { otherwise }
\end{array}\right. \\
i & =0,1, \ldots, N+1
\end{aligned}
$$

where $\omega\left(x_{i}\right)=\sin \frac{x-x_{i}}{2}, \phi\left(x_{i}\right)=\sin \frac{x_{i}-x}{2}, \theta=\sin \frac{h}{2} \sin h \sin \frac{3 h}{2}[31]$. The set $\left\{C_{0}(x), C_{1}(x), \ldots, C_{N+1}(x)\right\}$ forms a basis for the functions defined in the interval $[a, b]$.

Each $C_{i}(x)$ is twice continuously differentiable and the nonzero values of $C_{i}(x), C_{i}^{\prime}(x)$ and $C_{i}^{\prime \prime}(x)$ at the knots $x_{i}$ s can be computed from equation (7) as given in the Table 1 . 
Table 1: Values of $C_{i}(x)$ and its derivatives at knots

\begin{tabular}{l|l|l|l}
\hline \hline & $C_{i}\left(x_{i}\right)$ & $C_{i}^{\prime}\left(x_{i}\right)$ & $C_{i}^{\prime \prime}\left(x_{i}\right)$ \\
\hline \hline$x_{i-2}$ & 0 & 0 & 0 \\
\hline$x_{i-1}$ & $\sin ^{2}\left(\frac{h}{2}\right) \csc (h) \csc \left(\frac{3 h}{2}\right)$ & $\frac{3}{4} \csc \left(\frac{3 h}{2}\right)$ & $\frac{3(1+3 \cos (h)) \csc ^{2}\left(\frac{h}{2}\right)}{16\left[2 \cos \left(\frac{h}{2}\right)+\cos \left(\frac{3 h}{2}\right)\right]}$ \\
\hline$x_{i}$ & $\frac{2}{1+2 \cos (h)}$ & 0 & $\frac{-3 \cot ^{2}\left(\frac{3 h}{2}\right)}{2+4 \cos (h)}$ \\
\hline$x_{i+1}$ & $\sin ^{2}\left(\frac{h}{2}\right) \csc (h) \csc \left(\frac{3 h}{2}\right)$ & $-\frac{3}{4} \csc \left(\frac{3 h}{2}\right)$ & $\frac{3(1+3 \cos (h)) \csc ^{2}\left(\frac{h}{2}\right)}{16\left[2 \cos \left(\frac{h}{2}\right)+\cos \left(\frac{3 h}{2}\right)\right]}$ \\
\hline$x_{i+2}$ & 0 & 0 & 0 \\
\hline \hline
\end{tabular}

Let $U(x, t)$ and $V(x, t)$ be the approximate solutions to $u(x, t)$ and $v(x, t)$, respectively, defined as

$$
\begin{aligned}
& U(x, t)=\sum_{i=-1}^{N+1} \delta_{i} C_{i}(x), \\
& V(x, t)=\sum_{i=-1}^{N+1} \phi_{i} C_{i}(x)
\end{aligned}
$$

in which $\delta_{i}$ and $\phi_{i}$ are the time dependent parameters. The nodal values of the approximate solutions $U$ and $V$ and their first and second derivatives can be found from the (8) as

$$
\begin{array}{cl}
U_{i}=\alpha_{1} \delta_{i-1}+\alpha_{2} \delta_{i}+\alpha_{1} \delta_{i+1} & V_{i}=\alpha_{1} \phi_{i-1}+\alpha_{2} \phi_{i}+\alpha_{1} \phi_{i+1} \\
U_{i}^{\prime}=\beta_{1} \delta_{i-1}+\beta_{2} \delta_{i+1} & V_{i}^{\prime}=\beta_{1} \phi_{i-1}+\beta_{2} \phi_{i+1} \\
U_{i}^{\prime \prime}=\gamma_{1} \delta_{i-1}+\gamma_{2} \delta_{i}+\gamma_{1} \delta_{i+1} & V_{i}^{\prime \prime}=\gamma_{1} \phi_{i-1}+\gamma_{2} \phi_{i}+\gamma_{1} \phi_{i+1} \\
\alpha_{1}=\sin ^{2}\left(\frac{h}{2}\right) \csc (h) \csc \left(\frac{3 h}{2}\right) & \alpha_{2}=\frac{2}{1+2 \cos (h)} \\
\beta_{1}=-\frac{3}{4} \csc \left(\frac{3 h}{2}\right) & \beta_{2}=\frac{3}{4} \csc \left(\frac{3 h}{2}\right) \\
\gamma_{1}=\frac{3\left((1+3 \cos (h)) \csc ^{2}\left(\frac{h}{2}\right)\right)}{16\left(2 \cos \left(\frac{h}{2}\right)+\cos \left(\frac{3 h}{2}\right)\right)} & \gamma_{2}=-\frac{3 \cot ^{2}\left(\frac{h}{2}\right)}{2+4 \cos (h)}
\end{array}
$$

where $U_{i}$ and $V_{i}$ represent $U\left(x_{i}\right)$ and $V\left(x_{i}\right)$, respectively.

Substituting the approximate solutions (8) and their derivatives into (6) and rearranging the resulting equations by using nodal values of trigonometric B-spline functions gives the iterative system

$$
\begin{aligned}
& \kappa_{m 1} \delta_{m-1}^{n+1}+\kappa_{m 2} \phi_{m-1}^{n+1}+\kappa_{m 3} \delta_{m}^{n+1}+\kappa_{m 4} \phi_{m}^{n+1}+\kappa_{m 1} \delta_{m+1}^{n+1}+\kappa_{m 2} \phi_{m+1}^{n+1} \\
= & \kappa_{m 5} \delta_{m-1}^{n}+\kappa_{m 2} \phi_{m-1}^{n}+\kappa_{m 6} \delta_{m}^{n}+\kappa_{m 4} \phi_{m}^{n}+\kappa_{m 5} \delta_{m+1}^{n}+\kappa_{m 2} \phi_{m+1}^{n} \\
& \kappa_{m 2} \delta_{m-1}^{n+1}+\kappa_{m 7} \phi_{m-1}^{n+1}+\kappa_{m 4} \delta_{m}^{n+1}+\kappa_{m 8} \phi_{m}^{n+1}+\kappa_{m 2} \delta_{m+1}^{n+1}+\kappa_{m 7} \phi_{m+1}^{n+1} \\
= & \kappa_{m 2} \delta_{m-1}^{n}-\kappa_{m 7} \phi_{m-1}^{n}+\kappa_{m 4} \delta_{m}^{n}-\kappa_{m 8} \phi_{m}^{n}+\kappa_{m 2} \delta_{m+1}^{n}-\kappa_{m 7} \phi_{m+1}^{n}
\end{aligned}
$$


The coefficients of equation system (10) and (11) for KGE equation can be determined as follow

$$
\begin{aligned}
\kappa_{m 1} & =\left(-3 \varepsilon_{2} K^{2}-\varepsilon_{1}\right) \alpha_{1}-\gamma_{1} \\
\kappa_{m 2} & =\frac{2}{\Delta t} \alpha_{1} \\
\kappa_{m 3} & =\left(-3 \varepsilon_{2} K^{2}-\varepsilon_{1}\right) \alpha_{2}-\gamma_{2} \\
\kappa_{m 4} & =\frac{2}{\Delta t} \alpha_{2} \\
\kappa_{m 5} & =\left(\varepsilon_{1}-\varepsilon_{2} K^{2}\right) \alpha_{1}+\gamma_{1} \\
\kappa_{m 6} & =\left(\varepsilon_{1}-\varepsilon_{2} K^{2}\right) \alpha_{2}+\gamma_{2} \\
\kappa_{m 7} & =-\alpha_{1} \\
\kappa_{m 8} & =-\alpha_{2}
\end{aligned}
$$

where

$$
K=\alpha_{1} \delta_{i-1}^{n}+\alpha_{2} \delta_{i}^{n}+\alpha_{1} \delta_{i+1}^{n}
$$

The system (10) and (11) can be converted the following system in the matrix form

$$
\mathbf{A d}^{n+1}=\mathbf{B d}^{n}
$$

where

$$
\mathbf{A}=\left[\begin{array}{cccccccccc}
\kappa_{m 1} & \kappa_{m 2} & \kappa_{m 3} & \kappa_{m 4} & \kappa_{m 1} & \kappa_{m 2} & & & & \\
\kappa_{m 2} & \kappa_{m 7} & \kappa_{m 4} & \kappa_{m 8} & \kappa_{m 2} & \kappa_{m 7} & & & & \\
& & \kappa_{m 1} & \kappa_{m 2} & \kappa_{m 3} & \kappa_{m 4} & \kappa_{m 1} & \kappa_{m 2} & & \\
& & \kappa_{m 2} & \kappa_{m 7} & \kappa_{m 4} & \kappa_{m 8} & \kappa_{m 2} & \kappa_{m 7} & & \\
& & & \ddots & \ddots & \ddots & \ddots & \ddots & \ddots & \\
& & & & \kappa_{m 1} & \kappa_{m 2} & \kappa_{m 3} & \kappa_{m 4} & \kappa_{m 1} & \kappa_{m 2} \\
& & & & \kappa_{m 2} & \kappa_{m 7} & \kappa_{m 4} & \kappa_{m 8} & \kappa_{m 2} & \kappa_{m 7}
\end{array}\right]
$$

and

$$
\mathbf{B}=\left[\begin{array}{cccccccccc}
\kappa_{m 5} & \kappa_{m 2} & \kappa_{m 6} & \kappa_{m 4} & \kappa_{m 5} & \kappa_{m 2} & & & & \\
\kappa_{m 2} & -\kappa_{m 7} & \kappa_{m 4} & -\kappa_{m 8} & \kappa_{m 2} & -\kappa_{m 7} & & & & \\
& & \kappa_{m 5} & \kappa_{m 2} & \kappa_{m 6} & \kappa_{m 4} & \kappa_{m 5} & \kappa_{m 2} & & \\
& & \kappa_{m 2} & -\kappa_{m 7} & \kappa_{m 4} & -\kappa_{m 8} & \kappa_{m 2} & -\kappa_{m 7} & & \\
& & & \ddots & \ddots & \ddots & \ddots & \ddots & \ddots & \\
& & & & \kappa_{m 5} & \kappa_{m 2} & \kappa_{m 6} & \kappa_{m 4} & \kappa_{m 5} & \kappa_{m 2} \\
& & & & \kappa_{m 2} & -\kappa_{m 7} & \kappa_{m 4} & -\kappa_{m 8} & \kappa_{m 2} & -\kappa_{m 7}
\end{array}\right]
$$

The system $(12)$ consists of $2 N+2$ linear equations in $2 N+6$ unknown parameters $\mathbf{d}^{n+1}=\left(\delta_{-1}^{n+1}, \phi_{-1}^{n+1}, \delta_{0}^{n+1}, \phi_{0}^{n+1} \ldots, \delta_{N+1}^{n+1}, \phi_{N+1}^{n+1}\right)$. A unique solution of the system is dependent on the equal number of equations and parameters. Imposing the boundary conditions

$$
U_{x}(a, t)=0, U_{x}(b, t)=0, V_{x}(a, t)=0, V_{x}(b, t)=0
$$


gives a chance to reduce the number of parameters by generating relations between the parameters

$$
\delta_{-1}=\delta_{1}, \phi_{-1}=\phi_{1}, \delta_{N-1}=\delta_{N+1}, \phi_{N-1}=\phi_{N+1}
$$

Elimination of the parameters $\delta_{-1}, \phi_{-1}, \delta_{N+1}, \phi_{N+1}$ provides a solvable system having $2 N+2$ linear equations with $2 N+2$ unknown parameters. In the study, we solved this system by using adapted Thomas algorithm for the systems having six-banded coefficient matrices.

In order to initialize the iteration algorithm, we need the initial parameter vector $\mathbf{d}^{\mathbf{0}}$. Assuming $\mathbf{d}_{\mathbf{1}}^{\mathbf{0}}=\left(\delta_{-1}^{0}, \delta_{0}^{0}, . . \delta_{N}^{0}, \delta_{N+1}^{0}\right), \mathbf{d}_{\mathbf{2}}^{\mathbf{0}}=\left(\phi_{-1}^{0}, \phi_{0}^{0}, . . \phi_{N}^{0}, \phi_{N+1}^{0}\right)$ are the components of the initial vector $\mathbf{d}^{\mathbf{0}}$ of the iteration, we eliminate the parameters using the equalities

$$
\begin{aligned}
U_{x}(a, 0) & =0=\delta_{-1}^{0}-\delta_{1}^{0} \\
U_{x}\left(x_{i}, 0\right) & =\delta_{i-1}^{0}-\delta_{i+1}^{0}=U_{x}\left(x_{i}, 0\right), i=1, \ldots, N-1 \\
U_{x}(b, 0) & =0=\delta_{N-1}^{0}-\delta_{N+1}^{0}, \\
V_{x}(a, 0) & =0=\phi_{-1}^{0}-\phi_{1}^{0} \\
V_{x}\left(x_{i}, 0\right) & =\phi_{i-1}^{0}-\phi_{i+1}^{0}=V_{x}\left(x_{i}, 0\right), i=1, \ldots, N-1 \\
V_{x}(b, 0) & =0=\phi_{N-1}^{0}-\phi_{N+1}^{0}
\end{aligned}
$$

to be able to initalize the iteration 12 .

\section{$3 \quad$ Numerical Illustrations}

The validity and efficiency of the proposed method are checked by measuring the error between the numerical and the analytical solutions when exist using the discrete maximum error norms defined as

$$
L_{\infty}(t)=|u(x, t)-U(x, t)|_{\infty}=\max _{i}\left|u\left(x_{i}, t\right)-U\left(x_{i}, t\right)\right|
$$

at the time $t$.

The conservation laws computed by the numerical results representing energy $(\mathrm{E})$ and momentum(P) $[1,2,32$

$$
\begin{aligned}
& E=\frac{1}{2} \int_{-\infty}^{\infty} u_{t}^{2}+u_{x}^{2}-\varepsilon_{1} u^{2}-\frac{1}{2} \varepsilon_{2} u^{4} d x \\
& P=\int_{-\infty}^{\infty} u_{x} u_{t} d x
\end{aligned}
$$


for the nonlinear Klein-Gordon can also be also good indicators of the validity of the method even when the analytical solutions do not exist. The absolute relative changes $C\left(E_{t}\right)$ and $C\left(P_{t}\right)$ of conservation laws $E$ and $P$ are defined as

$$
\begin{aligned}
& C\left(E_{t}\right)=\left|\frac{E_{t}-E_{0}}{E_{0}}\right| \\
& C\left(P_{t}\right)=\left|\frac{P_{t}-P_{0}}{P_{0}}\right|
\end{aligned}
$$

where $E_{0}$ and $P_{0}$ are initial values of energy and momentum, respectively, as $E_{t}$ and $P_{t}$ are the computed values of these two laws at the time $t$.

\section{1 sech-type Single Solitary Wave}

The sech-type single solitary wave solution for the parameters $\varepsilon_{1}=2, \varepsilon_{2}=-1$ choice in the Klein-Gordon equation is given as

$$
u(x, t)=2 \operatorname{sech}(\sqrt{2}\{\sinh (1)\} x-\{\cosh (1)\} t)
$$

where sinh and cosh are known hyperbolic functions [33]. This solution represents a traveling wave of amplitude 2 whose peak is positioned at $x=0$ initially. As time passes, the wave propagates to the right along the $x$-axis. In order to accomplish the numerical simulation, the articifical problem interval is considered as $[-10,15]$. The initial condition is adapted from the analytical solution by substituting $t=0$ in the solution. The algorithm is run for various time and space step lengths up to the terminating time $t=3$. The propagation of the single solitary wave is simulated in Fig 1(a). The numerical results seem in a good agreement with theoretical aspects of the solution. The solitary propagates to the right along the horizontal axis without changing its shape and size. The maximum error accumulates at the peak of the solitary as expected, Fig 1(b). The discrete maximum norms computed at various times and the absolute relative changes computed at the simulation terminatig time $t=3$ are tabulated in Table 2.

When the space step length is chosen as 0.05, the maximum errors are determined in three decimal digits at $t=3$. Even though the decrease of the time step length improves the results, the decimal digit of the error does not change. The space step length $h=0.01$ and time step length $\Delta t=0.01$ generates the results with three decimal digit error at the simulation terminating time. Decrease of $\Delta t$ to 0.005 and 0.001 causes an improvement in the results and reduces the error to four decimal digits. One more decrease of the space step length to 0.005 generates three decimal digits accuracy in the results with $\Delta t=0.01$, four decimal digits 


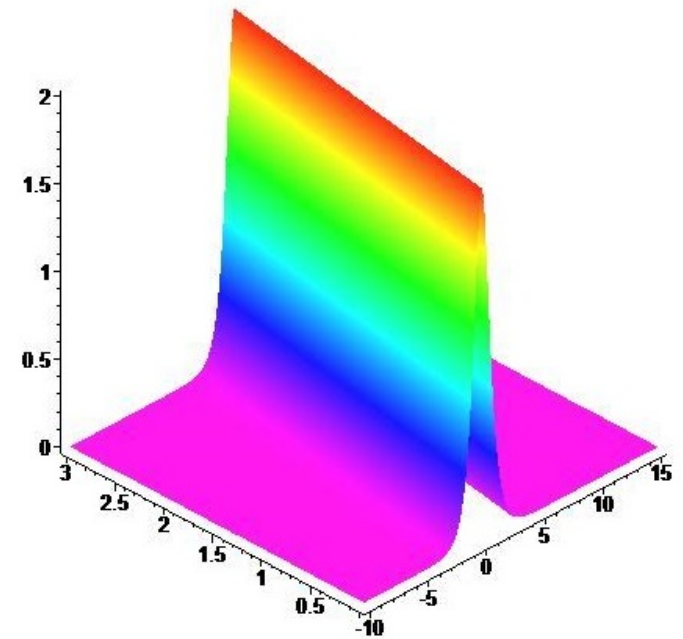

(a) Propagation of the single solitary wave

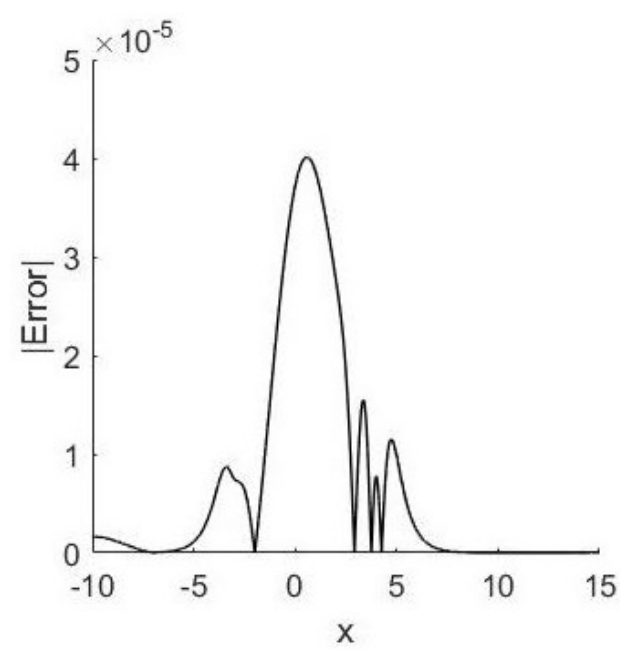

(b) The maximum error distribution

Figure 1: The simulation of propagation of the single solitary wave and the maximum error distribution at $t=3$ for $h=0.005$ and $\Delta t=0.001$

with $\Delta t=0.005$ at $t=3$. The choice of $\Delta t=0.001$ gives five decimal digit accurate solutions.

The initial values of the conservations laws are computed as $E_{0}=\frac{8}{3} \frac{\sqrt{2}\left(\cosh ^{2} 1-1\right)}{\sinh 1}=$ 4.431961243 and $P_{0}=-\frac{8}{3} \sqrt{2} \cosh 1=-5.819321497$ analytically. These laws are expected to be constant during the simulation as time goes. The conservation of both laws during the numerical simulation is a good indicator of an efficient algorithm, Table 2 .

The relative absolute change in the conservation law defining the momentum is less than $10^{-5}$ when $h=0.05$. The choice of $h$ as 0.01 causes six and seven decimal digit relative absolute changes with respect to the $\Delta t$ choices as 0.01 and 0.005 , respectively. The reduction of $\Delta t$ to 0.001 improves the relative absolute change to nine decimal digits.

When $h$ is reduced to 0.005 , the absolute relative changes of momentum are measured as $4.2916 \times 10^{-6}, 5.2841 \times 10^{-7}$ and $4.2749 \times 10^{-9}$ for the choices of the $\Delta t$ as $0.01,0.005$ and 0.001 respectively.

The absolute relative change of energy $C\left(E_{3}\right)$ is in six decimal digits at $t=3$ when $\Delta t=0.01$ for all choices of $h$ as $0.05,0.005$ and 0.001. Reducing the time step $\Delta t$ to 0.005 gives eight decimal digit absolute relative change when $h=0.05$. The absolute relative change when $h=0.01,0.005$ in seven decimal digits. When $\Delta t=0.001$, the absolute relative change is in eight decimal digits for $h=0.05$ and is in nine decimal digits for $h=0.01$ and $h=0.005$. 
Table 2: Discrete maximum norms and absolute relative changes of conservation laws at various times

\begin{tabular}{lllllll}
\hline \hline$h$ & $\Delta t$ & $L_{\infty}(1)$ & $L_{\infty}(2)$ & $L_{\infty}(3)$ & $C\left(P_{3}\right)$ & $C\left(E_{3}\right)$ \\
\hline 0.05 & 0.01 & $6.9995 \times 10^{-4}$ & $1.4660 \times 10^{-3}$ & $4.0116 \times 10^{-3}$ & $4.7628 \times 10^{-6}$ & $1.6394 \times 10^{-6}$ \\
& 0.005 & $8.8338 \times 10^{-4}$ & $1.7894 \times 10^{-3}$ & $3.2160 \times 10^{-3}$ & $2.6193 \times 10^{-6}$ & $9.9496 \times 10^{-8}$ \\
& 0.001 & $9.7707 \times 10^{-4}$ & $1.9321 \times 10^{-3}$ & $3.0102 \times 10^{-3}$ & $2.6159 \times 10^{-6}$ & $1.0035 \times 10^{-8}$ \\
0.01 & 0.01 & $3.5821 \times 10^{-4}$ & $5.1433 \times 10^{-4}$ & $1.5544 \times 10^{-3}$ & $4.2312 \times 10^{-6}$ & $2.4787 \times 10^{-6}$ \\
& 0.005 & $7.1150 \times 10^{-5}$ & $1.5002 \times 10^{-4}$ & $4.5035 \times 10^{-4}$ & $5.1616 \times 10^{-7}$ & $3.0131 \times 10^{-7}$ \\
& 0.001 & $3.5368 \times 10^{-5}$ & $7.1303 \times 10^{-5}$ & $1.2853 \times 10^{-4}$ & $7.5010 \times 10^{-9}$ & $2.0980 \times 10^{-9}$ \\
0.005 & 0.01 & $3.8598 \times 10^{-4}$ & $5.7203 \times 10^{-4}$ & $1.4977 \times 10^{-3}$ & $4.2916 \times 10^{-6}$ & $2.5055 \times 10^{-6}$ \\
& 0.005 & $8.9579 \times 10^{-5}$ & $1.2859 \times 10^{-4}$ & $3.8806 \times 10^{-4}$ & $5.2841 \times 10^{-7}$ & $3.0802 \times 10^{-7}$ \\
& 0.001 & $7.0113 \times 10^{-6}$ & $1.4656 \times 10^{-5}$ & $4.0086 \times 10^{-5}$ & $4.2749 \times 10^{-9}$ & $2.3658 \times 10^{-9}$ \\
\hline \hline
\end{tabular}

\section{2 tanh-type Traveling Wave}

In the second initial boundary value problem, we consider the case $\varepsilon_{1}=1, \varepsilon_{2}=-1$ in the nonlinear Klein-Gordon equation. This choice of the parameter provides an analytical solution of the form

$$
u(x, t)=\tanh \left(\frac{(x-c t)}{\sqrt{2\left(1-c^{2}\right)}}\right)
$$

where $c$ is the velocity of the traveling wave [34]. The existence of this real analytical solutions depends on the the condition $-1<c<1$. The wave travels along the $x$-axis to the right or to the left due to the sign of the velocity $c$. We choose the velocity $c=0.5$ for the convenience. The initial data for the numerical solution is generated from the analytical solution (17) by substituting $t=0$ into it. The homogenous Neumann conditions at both end of the finite interval $[-30,30]$ are used to complete the requirements of the iteration algorithm. The algorithm is run up to the terminating time $t=10$ with various values of the discretization parameters $h$ and $\Delta t$. The travel of the wave is simulated by the proposed algoritm successfully, Fig 2(a), The error takes its maximum values near points where the descent occurs as expected, Fig $(2(\mathrm{~b}))$.

The discrete maximum norms and the absolute relative changes of the conservation laws are computed for various values of the discretization parameters are tabularised in Table 3. The accuracy of the proposed method at the time $t=10$ is in three decimal digits when $h$ and $\Delta t$ are chosen as 0.2 and 0.05 , respectively. When the values of the discretization parameters are reduced to $h=0.1$ and $\Delta t=0.02$, the maximum error is measured as $4.4025 \times 10^{-4}$. Reducing the discretization parameters to $h=0.05$ and $\Delta t=0.01$ improves the accuracy of the 


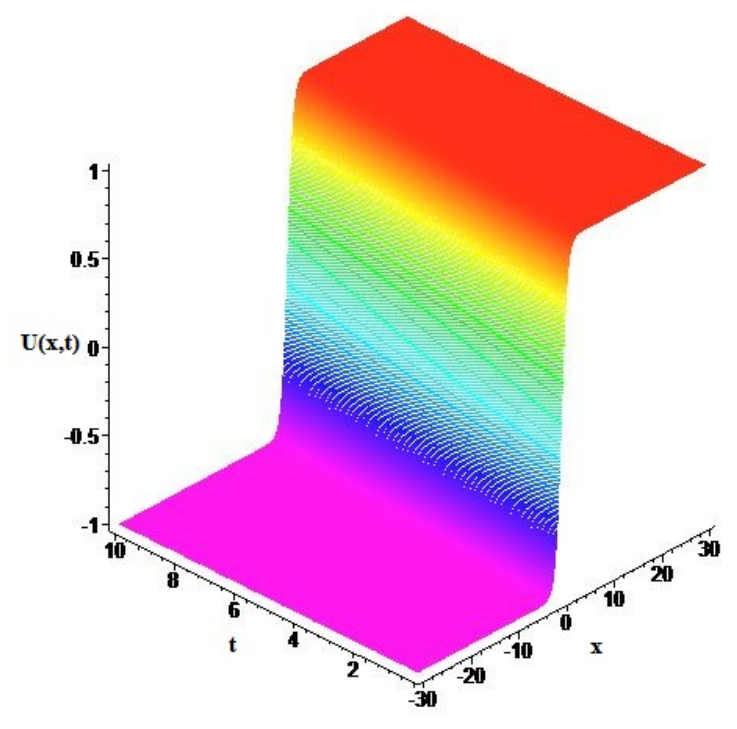

(a) Traveling wave simulation

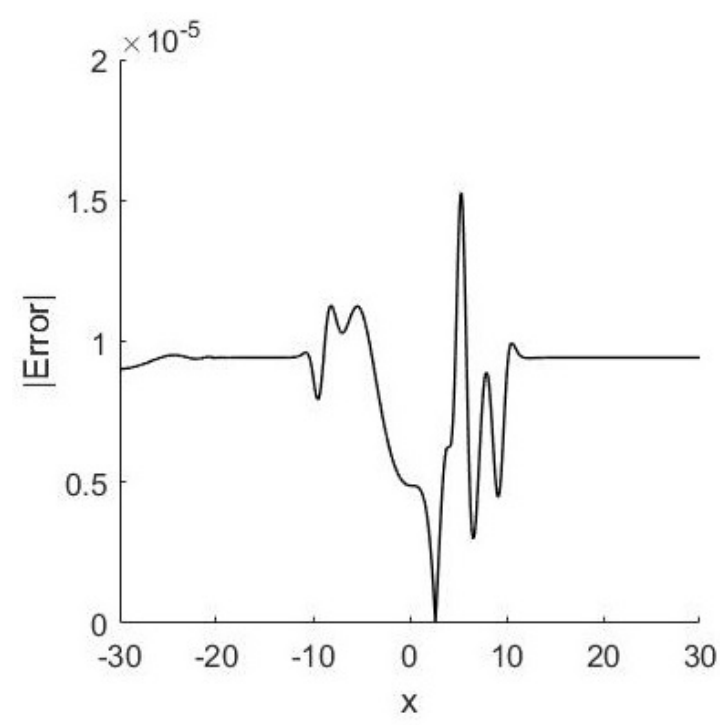

(b) The maximum error distribution

Figure 2: Traveling wave simulation and the maximum error distribution at $t=10$

results but not in decimal digits. Finally, the choice of the parameters as $h=0.02$ and $\Delta t=0.005$ gives a five decimal digit accuracy.

The initial values of the conservation laws describing the momentum and the energy are computed analytically as

$$
\begin{aligned}
E_{0} & =-\frac{1}{9} \frac{405 \mathrm{e}^{20 \sqrt{2} \sqrt{3}}+405 \mathrm{e}^{40 \sqrt{2} \sqrt{3}}+12 \mathrm{e}^{20 \sqrt{2} \sqrt{3}} \sqrt{2} \sqrt{3}+\tilde{A}}{1+3 \mathrm{e}^{20 \sqrt{2} \sqrt{3}}+3 \mathrm{e}^{40 \sqrt{2} \sqrt{3}}+\mathrm{e}^{60 \sqrt{2} \sqrt{3}}}=-13.91133789 \\
\tilde{A} & =4 \sqrt{2} \sqrt{3}-12 \mathrm{e}^{40 \sqrt{2} \sqrt{3}} \sqrt{2} \sqrt{3}-4 \mathrm{e}^{60 \sqrt{2} \sqrt{3}} \sqrt{2} \sqrt{3}+135 \mathrm{e}^{60 \sqrt{2} \sqrt{3}}+135 \\
P_{0} & =-2 / 9 \sqrt{2} \sqrt{3}=-0.5443310539
\end{aligned}
$$

One should note that the conservation law corresponding to the energy is integrated in the finite interval $[-30,30]$ for the convenience. The computed absolute relative changes for various values of the discretization parameters are indicators of the conservation of the energy and momentum successfully during the numerical simulation up to the time $t=10$, Table 3. The absolute relative change of the energy is bounded with at least six decimal digits with respect to the discretization parameter values used in the study. Similarly, the absolute relative change of the momentum is in five decimal digits. Reducing the discretization parameters 
to $h=0.02$ and $\Delta t=0.005$ improves the results to eleven decimal digits for the energy conservation and eight decimal digits for the momentum conservation law.

Table 3: Discrete maximum norms and absolute relative changes of conservation laws at various times

\begin{tabular}{lllll}
\hline \hline$h$ & $\Delta t$ & $L_{\infty}(10)$ & $C\left(E_{10}\right)$ & $C\left(P_{10}\right)$ \\
\hline 0.2 & 0.05 & $1.4255 \times 10^{-3}$ & $5.7598 \times 10^{-6}$ & $3.7187 \times 10^{-5}$ \\
0.1 & 0.02 & $4.4025 \times 10^{-4}$ & $3.6616 \times 10^{-7}$ & $2.3420 \times 10^{-6}$ \\
0.05 & 0.01 & $1.1098 \times 10^{-4}$ & $2.0256 \times 10^{-8}$ & $2.8785 \times 10^{-7}$ \\
0.02 & 0.005 & $1.5279 \times 10^{-5}$ & $3.5383 \times 10^{-11}$ & $3.5757 \times 10^{-8}$ \\
\hline \hline
\end{tabular}

\section{Conclusion}

The collocation method based on trigonometric cubic B-spline functions combined with the Crank-Nicolson implicit method proposed for the solutions of two initial boundary value problems defined for the nonlinear Klein-Gordon equation. The aasumption $v=u_{t}$ reduced the order of the equation to one and generated a coupled system of partial differential equations. The system is integrated in time by using the Crank-Nicolson method and fully discretized by using the collocation method. Adapting the boundary conditions and arranging the initial state give a linear equation system for the iteration.

The validity of the proposed method is invesitgated by solving two initial boundary value problems. The propagation of a positive single solitary and travel of a wave are simulated successfully by the proposed method. The error between the numerical and analytical solutions are measured by using the discrete maximum norm. The conservation laws defining the energy and the momentum of the system are also computed by using the numerical results. The absolute relative changes of the convervation laws are also good indicators of the accuracy and validity of the proposed method.

Acknowledgements: A brief part of this study was presented orally in International Conference on Applied Mathematics and Analysis, Ankara-Turkey, 2016. 


\section{References}

[1] Whitham, GB: Linear and Nonlinear Waves, John Wiley \& Sons, Newyork(1999).

[2] Debnath L: Nonlinear Partial Differential Equations for Scientists and Engineers, Birkhauser, Boston(2005).

[3] Detweiler, S: Klein-Gordon equation and rotating black holes. Phys. Rev. D, 22(10), 2323-2326 (1980).

[4] Galehouse, DC: Geometrical derivation of the Klein-Gordon equation. Int. J. Theor. Phys., 20(6), 457-479(1981).

[5] Weder, RA: Scattering theory for the Klein-Gordon equation. J. Func. Anal., 27(1), 100-117(1978).

[6] Lundberg, LE: Spectral and scattering theory for the Klein-Gordon equation. Commun. Math. Phys., 31(3), 243-257(1973).

[7] Ablowitz, MJ:Nonlinear Dispersive Waves, Cambridge University Press, Cambridge(2011).

[8] Sharma, AS, \& Buti, B: Envelope solitons and holes for sine-Gordon and non-linear Klein-Gordon equations. J. Phys. A-Math. Gen., 9(11), 18231826(1976)..

[9] Dusuel, S, Michaux, P, \& Remoissenet, M: From kinks to compactonlike kinks. Phys. Rev. E., 57(2), 2320-2326(1998).

[10] Kim, JJ, \& Hong, WP: New solitary-wave solutions for the generalized reaction Duffing model and their dynamics. Z. Naturforsch. Pt. A, 59(11), 721-728 (2004).

[11] Burt, PB (1974). Solitary waves in nonlinear field theories. Physical Review Letters, 32(19), 1080.

[12] Huang, D. J., \& Zhang, H. Q. (2005). The extended first kind elliptic subequation method and its application to the generalized reaction Duffing model. Physics Letters A, 344(2), 229-237.

[13] Akter, J., \& Akbar, M. A. (2015). Exact solutions to the Benney-Luke equation and the Phi-4 equations by using modified simple equation method. Results in Physics, 5, 125-130. 
[14] Chambers, L. G. (1966). Derivation of solutions of the Klein-Gordon equation from solutions of the wave equation. Proceedings of the Edinburgh Mathematical Society (Series 2), 15(02), 125-129.

[15] Fleischer, W., \& Soff, G. (1984). Bound state solutions of the Klein-Gordon equation for strong potentials. Zeitschrift f'ur Naturforschung A, 39(8), 703719 .

[16] Burt, P. B., \& Reid, J. L. (1976). Exact solution to a nonlinear Klein-Gordon equation. Journal of Mathematical Analysis and Applications, 55(1), 43-45.

[17] Kudryavtsev, A. E. (1975). Solitonlike solutions for a Higgs scalar field. Institute of Theoretical and Experimental Physics.

[18] Ablowitz, M. J., Kruskal, M. D., \& Ladik, J. F. (1979). Solitary wave collisions. SIAM Journal on Applied Mathematics, 36(3), 428-437.

[19] Campbell, D. K., \& Peyrard, M. (1986). Solitary wave collisions revisited. Physica D: Nonlinear Phenomena, 18(1), 47-53.

[20] Strauss, W., \& Vazquez, L. (1978). Numerical solution of a nonlinear KleinGordon equation. Journal of Computational Physics, 28(2), 271-278.

[21] Jiménez, S., \& Vázquez, L. (1990). Analysis of four numerical schemes for a nonlinear Klein-Gordon equation. Applied Mathematics and Computation, 35(1), 61-94.

[22] Dehghan, M., \& Shokri, A. (2009). Numerical solution of the nonlinear KleinGordon equation using radial basis functions. Journal of Computational and Applied Mathematics, 230(2), 400-410.

[23] Sarboland, M., \& Aminataei, A. (2015). Numerical solution of the nonlinear Klein-Gordon equation using multiquadric quasi-interpolation scheme. Univ J Appl Math, 3(3), 40-49.

[24] Cao, W. M., \& Guo, B. Y. (1993). Fourier collocation method for solving nonlinear Klein-Gordon equation. Journal of Computational Physics, 108(2), 296-305.

[25] Rashidinia, J., Ghasemi, M., \& Jalilian, R. (2010). Numerical solution of the nonlinear Klein-Gordon equation. Journal of Computational and Applied Mathematics, 233(8), 1866-1878. 
[26] Zahra, W. K., Ouf, W. A., \& El-Azab, M. S. (2016). A robust uniform Bspline collocation method for solving the generalized PHI-four equation. Applications and Applied Mathematics, 11(1), 384-396.

[27] Dag, I., \& Ersoy, O. (2016). The exponential cubic B-spline algorithm for Fisher equation. Chaos, Solitons \& Fractals, 86, 101-106.

[28] Ersoy, O., \& Dag, I. (2015). Numerical solutions of the reaction diffusion system by using exponential cubic B-spline collocation algorithms. Open Physics, $13(1)$.

[29] Korkmaz, A., \& Dag, I. (2013). Cubic B-spline differential quadrature methods and stability for Burgers' equation. Engineering Computations, 30(3), $320-344$.

[30] Korkmaz, A., \& Dag, I. (2012). Cubic B-spline differential quadrature methods for the advection-diffusion equation. International Journal of Numerical Methods for Heat \& Fluid Flow, 22(8), 1021-1036.

[31] Abbas, M., Majid, A. A., Ismail, A. I. M., \& Rashid, A. (2014, May). Numerical method using cubic trigonometric B-spline technique for nonclassical diffusion problems. In Abstract and applied analysis (Vol. 2014). Hindawi Publishing Corporation.

[32] Jhangeer, A., \& Sharif, S. (2014). Conserved quantities for the non-linear Klein-Gordon equation. Afrika Matematika, 25(3), 833-840.

[33] Polyanin, A. D., \& Zaitsev, V. F. (2004). Handbook of nonlinear partial differential equations. CRC press.

[34] Zaki, S.I., Gardner L.R.T., Gardner G.A., 1997, Numerical simulations of Klein-Gordon solitary wave interactions Il Nuovo Cimento, 112B, N.7. 